\title{
A Note on Representations of Linear Inequalities in Non-Convex Mixed-Integer Quadratic Programs
}

\author{
Adam N. Letchford* Daniel J. Grainger ${ }^{\dagger}$ \\ To appear in Operations Research Letters
}

\begin{abstract}
In the literature on the quadratic 0-1 knapsack problem, several alternative ways have been given to represent the knapsack constraint in the quadratic space. We extend this work by constructing analogous representations for arbitrary linear inequalities for arbitrary nonconvex mixed-integer quadratic programs with bounded variables.
\end{abstract}

\section{Introduction}

It has long been known that one can obtain useful reformulations of $\mathcal{N} \mathcal{P}$ hard optimisation problems by introducing additional variables representing products or squares of original variables. This idea has been applied to, e.g., 0-1 linear programs [18, 22], 0-1 quadratic programs [1, 11, 19], non-convex quadratic programs $[7,23]$, non-convex quadratically constrained quadratic programs $[12,21]$, and many other problems.

The presence of additional variables leads to some flexibility in the choice of representing linear constraints. In their paper on semidefinite programming relaxations of the 0-1 quadratic knapsack problem (QKP), Helmberg et al. [16] consider three different representations of the knapsack constraint. In his thesis, Helmberg [14], considers also a fourth representation. The purpose of this note is to extend this work, by constructing analogous representations for arbitrary linear inequalities for arbitrary mixed-integer quadratic programs (MIQPs). The only restriction is that all variables involved in the inequality must be explicitly bounded.

The paper has a very simple structure. The literature is reviewed in Section 2, and the new results are in Section 3.

\footnotetext{
* Corresponding author. Department of Management Science, Lancaster University, Lancaster LA1 4YW, UK. E-mail: a.n.letchford@lancaster.ac.uk

${ }^{\dagger}$ Former PhD student. E-mail: grainged@gmail.com
} 


\section{Literature Review}

We now give a brief overview of the relevant literature. We cover general quadratic 0-1 programs in Subsection 2.1, the QKP in Subsection 2.2, and bounded MIQP in Subsection 2.3.

\subsection{Quadratic 0-1 programming}

A quadratic 0-1 program (0-1 QP) with $n$ variables and $m$ constraints is a problem of the form

$$
\max \left\{x^{T} Q x: A x \leq b, x \in\{0,1\}^{n}\right\},
$$

where $Q=\left\{q_{i j}\right\} \in \mathbb{Q}^{n \times n}, A \in \mathbb{Q}^{m \times n}$ and $b \in \mathbb{Q}^{m}$. It is well known that 0-1 QPs are strongly $\mathcal{N} \mathcal{P}$-hard, yet have many important applications (see, e.g., $[4,10])$.

One can convert 0-1 QPs into 0-1 linear programs using a standard linearisation trick, due to Fortet [11]. We introduce for all $1 \leq i<j \leq n$ the binary variable $x_{i j}$, representing the product $x_{i} x_{j}$. To simplify notation, we identify $x_{j i}$ with $x_{i j}$. The $0-1$ QP is then formulated as:

$$
\begin{array}{ccl}
\max & \sum_{1 \leq i \leq n} q_{i i} x_{i}+\sum_{1 \leq i<j \leq n}\left(q_{i j}+q_{j i}\right) x_{i j} & \\
\text { s.t. } & A x \leq b & \\
& x_{i j} \leq x_{i} & (1 \leq i \leq n, j \neq i) \\
x_{i}+x_{j} \leq x_{i j}+1 & (1 \leq i<j \leq n) \\
x_{i} \in\{0,1\}^{n} & (1 \leq i \leq n) \\
x_{i j} \in\{0,1\}^{n} & (1 \leq i<j \leq n) .
\end{array}
$$

Unfortunately, the LP relaxation of this formulation typically gives a very weak upper bound. To strengthen it, one can use various families of cutting planes, such as those presented in $[1,10,19]$.

An alternative way to construct a non-trivial relaxation is to use semidefinite programming (SDP); see, e.g., [15, 20]. The basic idea is as follows. Define the matrix $X=x x^{T}$, along with the augmented matrix

$$
X^{+}=\left(\begin{array}{l}
1 \\
x
\end{array}\right)\left(\begin{array}{l}
1 \\
x
\end{array}\right)^{T}=\left(\begin{array}{cc}
1 & x^{T} \\
x & X
\end{array}\right) .
$$

Clearly, $X^{+}$will be real, symmetric and positive semidefinite in a feasible 0-1 QP solution. Moreover, for binary $x_{i}$ we have $x_{i}=x_{i}^{2}$, meaning that the main diagonal of $X$ should be equal to $x$. This leads to the SDP relaxation

$$
\max \left\{Q \bullet X: A x \leq b, \operatorname{diag}(X)=x, X^{+} \in \mathcal{S}_{n+1}^{+}\right\},
$$

where ' $\bullet$ ' denotes inner product and ' $\mathcal{S}_{n+1}^{+}$' denotes the cone of psd matrices of order $n+1$. For ways to strengthen this relaxation, see again $[15,20]$. 
Finally, one can instead use Lagrangian relaxations (e.g., Caprara [8]) or convex $Q P$ relaxations (e.g., Billionnet et al. [3]). We omit details, for brevity.

\subsection{The quadratic knapsack problem}

The quadratic knapsack problem (QKP) is a special kind of 0-1 QP in which there is a single linear constraint with non-negative coefficients. A QKP instance takes the form:

$$
\max \left\{x^{T} Q x: w^{T} x \leq c, x \in\{0,1\}^{n}\right\},
$$

where $w \in \mathbb{Z}_{+}^{n}$ is the vector of item weights and $c \in \mathbb{Z}_{+}$is the knapsack capacity. We refer the reader to $[9,17]$ for excellent surveys on the QKP.

Helmberg et al. [16] explored three natural SDP relaxations of the QKP. The simplest, called 'SQK1', is:

$$
\max \left\{Q \bullet X: w^{T} x \leq c, \operatorname{diag}(X)=x, X^{+} \in \mathcal{S}_{n+1}^{+}\right\} .
$$

The second, called 'SQK2', is obtained by replacing the knapsack constraint $w^{T} x \leq c$ with the constraint $w^{T} X w \leq c^{2}$. The third, 'SQK3', is obtained by adding the following valid inequalities to SQK1:

$$
\sum_{i \neq j} w_{i} x_{i j} \leq\left(c-w_{j}\right) x_{j} \quad(j=1, \ldots, n) .
$$

These latter inequalities can be derived from the original knapsack constraint by applying the well-known reformulation-linearization technique (RLT) of Adams \& Sherali [1, 22].

Helmberg et al. prove that SQK3 dominates SQK2, which in turns dominates SQK1. In his thesis [14], Helmberg mentions a fourth SDP relaxation, which he attributes to Bauvin and Goemans. It is obtained by replacing the original knapsack constraint with $w^{T} X w \leq c\left(w^{T} x\right)$. We will call this relaxation 'SQK2 ${ }^{+}$'. Helmberg shows that $\mathrm{SQK}^{+}$is intermediate in strength between SQK2 and SQK3.

A comprehensive computational study of all four SDP relaxations is given in [17]. It turns out that SQK2 ${ }^{+}$gives the best compromise between upper bound strength and computing time, since it typically gives a bound of similar quality to that of SQK3, but with much less computing effort, due to the smaller number of linear constraints.

\subsection{Mixed-integer quadratic programs}

Now we turn our attention to bounded MIQPs, which include 0-1 QPs as a special case. A bounded MIQP takes the form:

$$
\max \left\{x^{T} Q x+c^{T} x: A x \leq b, 0 \leq x \leq u, x \in \mathbb{R}_{+}^{n}, x_{i} \in \mathbb{Z}(i \in I)\right\},
$$


where $I \subset\{1, \ldots, n\}$ and $u \in \mathbb{Q}_{+}^{n}$. (Of course, one can assume w.l.o.g. that $u_{i} \in \mathbb{Z}_{+}$for all $i \in I$.) Bounded MIQPs are $\mathcal{N} \mathcal{P}$-hard in general, and are especially challenging when $Q$ is not psd (i.e., when the objective function is non-convex).

A natural SDP relaxation of the MIQP is:

$$
\max \left\{Q \bullet X+c^{T} x: A x \leq b, 0 \leq x \leq u, X^{+} \in \mathcal{S}_{n+1}^{+}\right\} .
$$

Note that this relaxation does not exploit integrality. Moreover, it is unbounded in general, since the entries $X_{i i}$ can be increased without limit. To make it bounded, one can add the upper bounds $X_{i i} \leq u_{i}^{2}$. Or, even better, as pointed out by Anstreicher [2], one can add the constraints

$$
X_{i i} \leq u_{i} x_{i} \quad(i=1, \ldots, n) .
$$

The relaxation can be strengthened further by adding various valid inequalities, some of which exploit integrality; see, e.g., [5, 6, 13].

\section{Main Results}

Consider a non-convex MIQP of the form (1), and let $\alpha^{T} x \leq \beta$ be one of the linear constraints in the system $A x \leq b$. We define the sets

$$
\begin{aligned}
& N=\{1, \ldots, n\} \\
& N^{+}=\left\{i \in N: \alpha_{i}>0\right\} \\
& N^{-}=\left\{i \in N: \alpha_{i}<0\right\}
\end{aligned}
$$

and the quantities

$$
U=\sum_{i \in N^{+}} \alpha_{i} u_{i}, \quad L=\sum_{i \in N^{-}} \alpha_{i} u_{i} .
$$

We assume that $L<\beta<U$, since otherwise the linear constraint would be implied by the bounds $0 \leq x \leq u$.

Now, by definition, if a pair $(x, X)$ represents a feasible MIQP solution, we must have $L \leq \alpha^{T} x \leq \beta$ and

$$
\left(\alpha^{T} x\right)^{2}=\left(\alpha^{T} x\right)\left(x^{T} \alpha\right)=\alpha^{T}\left(x x^{T}\right) \alpha=\alpha^{T} X \alpha .
$$

Thus, if we project the set of feasible MIQP solutions in $(x, X)$-space onto a 2-dimensional subspace having $\alpha^{T} x$ and $\alpha^{T} X \alpha$ as axes, it must be contained inside the convex region represented in Figure 1. One can check that this region is defined by the convex quadratic inequality

$$
\alpha^{T} X \alpha \geq\left(\alpha^{T} x\right)^{2}
$$

and the linear inequality

$$
\alpha^{T} X \alpha \leq(\beta+L) \alpha^{T} x-\beta L .
$$

We have the following result: 


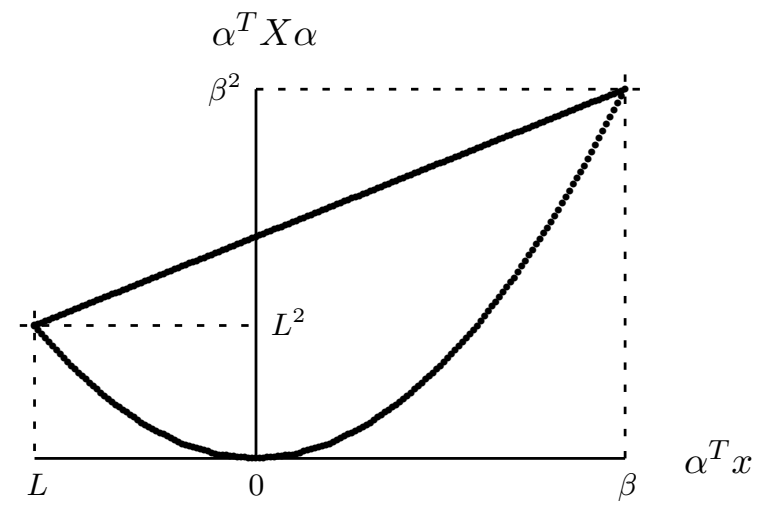

Figure 1: Projection of feasible region onto two-dimensional subspace.

Theorem 1 Consider the SDP relaxation of the MIQP obtained by adding the constraints (3) to (2). This relaxation satisfies (4). It does not in general satisfy (5), but it does satisfy the weaker inequality

$$
\alpha^{T} X \alpha \leq(U+L) \alpha^{T} x-U L
$$

(see Figure 2).

Proof. From the well-known concept of Schur complement, $X^{+} \in \mathcal{S}_{n+1}^{+}$if and only if $X-x x^{T} \in \mathcal{S}_{n}^{+}$. This implies that $\alpha^{T}\left(X-x x^{T}\right) \alpha \geq 0$, i.e., that the SDP relaxation satisfies (4).

We now define vectors $x^{L}, x^{U} \in \mathbb{Q}_{+}^{n}$, as follows. We let $x_{i}^{L}=u_{i}$ for all $i \in N^{-}$and $x_{i}^{L}=0$ for all $i \in N \backslash N^{-}$. Similarly, we let $x_{i}^{U}=u_{i}$ for all $i \in N^{+}$and $x_{i}^{U}=0$ for all $i \in N \backslash N^{+}$. We also define the corresponding matrices $X^{L}=x^{L}\left(x^{L}\right)^{T}$ and $X^{U}=x^{U}\left(x^{U}\right)^{T}$. (Note that $\alpha^{T} x^{L}=L$ and $\alpha^{T} x^{U}=U>\beta$. As a result, the pair $\left(x^{L}, X^{L}\right)$ is feasible for the relaxation, but the pair $\left(x^{U}, X^{U}\right)$ is not.) Now, consider the convex combination

$$
(\tilde{x}, \tilde{X})=\frac{U-\beta}{U-L}\left(x^{L}, X^{L}\right)+\frac{\beta-L}{U-L}\left(x^{U}, X^{U}\right) .
$$

One can check that $\alpha^{T} \tilde{x}=\beta$ and $\alpha^{T} \tilde{X} \alpha=\beta L+U(\beta-L)$. Thus, $(\tilde{x}, \tilde{X})$ is feasible for the relaxation, but violates the inequality (5).

All that remains is to show that the relaxation satisfies (6). We will do this in three steps. First, for any $\{i, j\} \subset N$, consider the following principle submatrix of $X^{+}$:

$$
\left(\begin{array}{ll}
X_{i i} & X_{i j} \\
X_{i j} & X_{j j}
\end{array}\right)
$$

Since this submatrix must be psd, it follows that the relaxation satisfies

$$
\left(\begin{array}{ll}
u_{j} & -u_{i}
\end{array}\right)\left(\begin{array}{cc}
X_{i i} & X_{i j} \\
X_{i j} & X_{j j}
\end{array}\right)\left(\begin{array}{c}
u_{j} \\
-u_{i}
\end{array}\right) \geq 0
$$


or, equivalently, $2 u_{i} u_{j} X_{i j} \leq u_{j}^{2} X_{i i}+u_{i}^{2} X_{j j}$. Together with (3), this implies $2 u_{i} u_{j} X_{i j} \leq u_{i} u_{j}^{2} x_{i}+u_{i}^{2} u_{j} x_{j}$, or, equivalently,

$$
2 X_{i j} \leq u_{j} x_{i}+u_{i} x_{j}
$$

Second, consider the following principle submatrix:

$$
\left(\begin{array}{ccc}
1 & x_{i} & x_{j} \\
x_{i} & X_{i i} & X_{i j} \\
x_{j} & X_{i j} & X_{j j}
\end{array}\right)
$$

We have

$$
\left(\begin{array}{lll}
-u_{i} u_{j} & u_{j} & u_{i}
\end{array}\right)\left(\begin{array}{ccc}
1 & x_{i} & x_{j} \\
x_{i} & X_{i i} & X_{i j} \\
x_{j} & X_{i j} & X_{j j}
\end{array}\right)\left(\begin{array}{c}
-u_{i} u_{j} \\
u_{j} \\
u_{i}
\end{array}\right) \geq 0
$$

or, equivalently,

$$
2 u_{i} u_{j} X_{i j} \geq 2 u_{i} u_{j}^{2} x_{i}+2 u_{i}^{2} u_{j} x_{j}-u_{j}^{2} X_{i i}-u_{i}^{2} X_{j j}-\left(u_{i} u_{j}\right)^{2} .
$$

Together with (3), this implies:

$$
2 u_{i} u_{j} X_{i j} \geq u_{i} u_{j}^{2} x_{i}+u_{i}^{2} u_{j} x_{j}-\left(u_{i} u_{j}\right)^{2},
$$

or, equivalently,

$$
2 X_{i j} \geq u_{j} x_{i}+u_{i} x_{j}-u_{i} u_{j}
$$

Finally, the inequalities (7) imply

$$
\alpha_{i} \alpha_{j} X_{i j} \leq \alpha_{i} \alpha_{j}\left(u_{j} x_{i}+u_{i} x_{j}\right) / 2
$$

when $\alpha_{i} \alpha_{j}>0$, and the inequalities (8) imply

$$
\alpha_{i} \alpha_{j} X_{i j} \leq \alpha_{i} \alpha_{j}\left(u_{j} x_{i}+u_{i} x_{j}\right) / 2-\left(\alpha_{i} u_{i}\right)\left(\alpha_{j} u_{j}\right)
$$

when $\alpha_{i} \alpha_{j}<0$. Summing the inequalities (9) for all (not necessarily distinct) pairs $i, j$ with $\alpha_{i} \alpha_{j}>0$, together with the inequalities (10) for all pairs $\{i, j\}$ with $\alpha_{i} \alpha_{j}<0$, yields (6).

We are now in a position to define our analogues of SQK1, SQK2, SQK2 ${ }^{+}$ and SKQ3 for bounded MIQPs. The analogue of SQK1 is obtained simply by taking the SDP (2) and adding the constraints (3). The analogue of SQK2 is obtained from SQK1 by taking each constraint $\alpha^{T} x \leq \beta$ in the system $A x \leq b$ and replacing it with the constraint $\alpha^{T} X \alpha \leq \beta^{2}$. (This relaxation is however valid only if (i) $b>0$ and (ii) all feasible MIQP solutions satisfy $A x \geq-b$.) The analogue of $\mathrm{SQK} 2^{+}$is obtained by replacing each constraint $\alpha^{T} x \leq \beta$ with the stronger constraint (5). As for the analogue of SQK3, this is obtained by replacing each constraint $\alpha^{T} x \leq \beta$ with the $2 n$ constraints presented in the following proposition. 


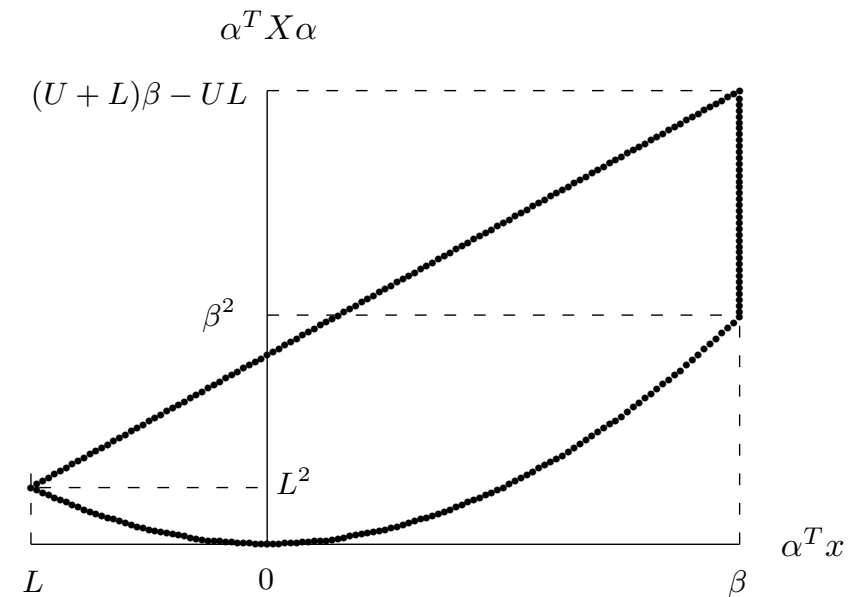

Figure 2: Projection of SDP relaxation onto same subspace.

Proposition 1 Let $\alpha^{T} x \leq \beta$ be one of the constraints in the system $A x \leq$ $b$. The following $2 n$ linear inequalities are valid for all pairs $(x, X)$ that represent feasible MIQP solutions:

$$
\begin{array}{cc}
\sum_{j \in N} \alpha_{j} X_{i j} \leq \beta x_{i} & (i \in N) \\
u_{i} \alpha^{T} x-\sum_{j \in N} \alpha_{j} X_{i j} \leq \beta\left(u_{i}-x_{i}\right) & (i \in N) .
\end{array}
$$

Collectively, they dominate the inequality (5).

Proof. Given that $\alpha^{T} x \leq \beta$ and $x \geq 0$, we have the quadratic inequality $\left(\beta-\alpha^{T} x\right) x_{i} \geq 0$ for all $i \in N$, which implies (11). Similarly, given that $x \leq u$, we have the quadratic inequality $\left(\beta-\alpha^{T} x\right)\left(u_{i}-x_{i}\right) \geq 0$ for all $i \in N$, which implies (12).

Now, if we take the inequality (11) for some $i \in N^{+}$and multiply it by $\alpha_{i}$, we obtain

$$
\alpha_{i} \sum_{j \in N} \alpha_{j} X_{i j} \leq \beta \alpha_{i} x_{i}
$$

On the other hand, if we take the inequality (12) for some $i \in N^{-}$and multiply it by $\alpha_{i}$, we obtain

$$
\alpha_{i} \sum_{j \in N} \alpha_{j} X_{i j} \leq \beta \alpha_{i} x_{i}+\left(\alpha_{i} u_{i}\right) \alpha^{T} x-\beta\left(\alpha_{i} u_{i}\right) .
$$

Summing up (13) over all $i \in N^{+}$and (14) over all $i \in N^{-}$, we obtain (5).

Note that the proof of Proposition 1 actually implies that it suffices to replace each original constraint with only $n$ constraints rather than $2 n$. 
We end the paper with a final remark. The linear inequality (5) remains valid if we replace $L$ with:

$$
\min \left\{\alpha^{T} x: A x \leq b, 0 \leq x \leq u, x_{i} \in \mathbb{Z}(i \in I)\right\},
$$

or indeed any lower bound on the value taken by $\alpha^{T} x$ in a feasible solution. In this way, one can strengthen our analogue of $\mathrm{SQK} 2^{+}$. It is not clear whether one could strengthen our analogue of SQK3 using a similar argument, since the coefficient $L$ does not appear explicitly in the constraints (11) and (12).

Acknowledgement: The research of the first author was partially supported by the Engineering and Physical Sciences Research Council under grant EP/D072662/1.

\section{References}

[1] W.P. Adams \& H.D. Sherali (1986) A tight linearization and an algorithm for zero-one quadratic programming problems. Mgmt. Sci., 32, 1274-1290.

[2] K. Anstreicher (2009) Semidefinite programming versus the reformulation-linearization technique for nonconvex quadratically constrained quadratic programming. J. Glob. Optim., 43, 471-484.

[3] A. Billionnet, S. Elloumi \& M.-C. Plateau (2009) Improving the performance of standard solvers for quadratic $0-1$ programs by a tight convex reformulation: the QCR method. Discr. Appl. Math., 157, 1185-1197.

[4] E. Boros \& P. Hammer (2002) Pseudo-boolean optimization. Discr. Appl. Math., 123, 155-225.

[5] S. Burer \& A.N. Letchford (2014) Unbounded convex sets for nonconvex mixed-integer quadratic programming. Math. Program., 143, $231-256$.

[6] S. Burer \& A. Saxena (2011) The MILP road to MIQCP. In J. Lee \& S. Leyffer (eds.) Mixed Integer Nonlinear Programming, pp. 373-406. New York: Springer US.

[7] S. Burer \& D. Vandenbussche (2008) A finite branch-and-bound algorithm for nonconvex quadratic programming via semidefinite relaxations. Math. Program., 113, 259-282.

[8] A. Caprara (2008) Constrained 0-1 quadratic programming: basic approaches and extensions. Eur. J. Oper. Res., 187, 1494-1503. 
[9] A. Caprara, D. Pisinger \& P. Toth (1999) Exact solution of the quadratic knapsack problem. INFORMS J. Comput. 11, 125-137.

[10] M.M. Deza \& M. Laurent (1997) Geometry of Cuts and Metrics. Berlin: Springer-Verlag.

[11] R. Fortet (1959) L'Algèbre de Boole et ses applications en recherche opérationnelle. Cahiers Centre Etudes Rech. Oper., 1, 5-36.

[12] T. Fujie \& M. Kojima (1997) Semidefinite programming relaxation for nonconvex quadratic programs. J. Glob. Optim., 10, 367-380.

[13] L. Galli, K. Kaparis \& A.N. Letchford (2011) Gap inequalities for nonconvex mixed-integer quadratic programs. Oper. Res. Lett., 39, 297300 .

[14] C. Helmberg (2000) Semidefinite Programming for Combinatorial Optimization. Habilitationsschrift, Technical University of Berlin.

[15] C. Helmberg \& F. Rendl (1998) Solving quadratic (0,1)-programs by semidefinite programs and cutting planes. Math. Program., 82, 291-315.

[16] C. Helmberg, F. Rendl \& R. Weismental (2000) A semidefinite programming approach to the quadratic knapsack problem. J. Combin. Optim., 4, 197-215.

[17] H. Kellerer, U. Pferschy \& D. Pisinger (2004) Knapsack Problems. Berlin: Springer-Verlag.

[18] L. Lovász \& A.J. Schrijver (1991) Cones of matrices and set-functions and 0-1 optimization. SIAM J. Optim., 1, 166-190.

[19] M.W. Padberg (1989) The Boolean quadric polytope: some characteristics, facets and relatives. Math. Program., 45, 139-172.

[20] S. Poljak, F. Rendl \& H. Wolkowicz (1995) A recipe for semidefinite relaxation for (0,1)-quadratic programming. J. Glob. Optim., 7, 51-73.

[21] M. Ramana (1993) An Algorithmic Analysis of Multiquadratic and Semidefinite Programming Problems. PhD Thesis, Johns Hopkins University, Baltimore, MD.

[22] H.D. Sherali \& W.P. Adams (1990) A hierarchy of relaxations between the continuous and convex hull representations for zero-one programming problems. SIAM J. Discr. Math., 3, 411-430.

[23] H.D. Sherali \& C.H. Tuncbilek (1995) A reformulation-convexification approach for solving nonconvex quadratic programming problems. $J$. Glob. Optim., 7, 1-31. 